\title{
Induction of adipose tissue regeneration by chemically-modified hyaluronic acid
}

\author{
Nicholas P. Rhodes* \\ Division of Clinical Engineering, \\ UK Centre for Tissue Engineering, \\ University of Liverpool, \\ Duncan Building, Daulby Street, \\ Liverpool L69 3GA, UK \\ Fax: +44-151-706-4915 \\ E-mail: npr@liv.ac.uk \\ ${ }^{*}$ Corresponding author
}

\section{Chiara di Bartolo and Davide Bellini}

Fidia Advanced Biopolymers, via Ponte della Fabbrica 3/A,

Abano Terme, Padova 35031, Italy

\section{John A. Hunt}

Division of Clinical Engineering,

UK Centre for Tissue Engineering,

University of Liverpool,

Duncan Building, Daulby Street,

Liverpool L69 3GA, UK

\begin{abstract}
Hyaluronan was modified by amidation of the carboxyl groups with dodycyl amides to produce a viscous gel (HYADD3). Small aliquots were implanted intramuscularly in Wistar rats at a concentration of $30 \mathrm{mg} / \mathrm{ml}$ for up to 12 weeks. Standard histology and immunohistochemistry demonstrated low levels of inflammation at all time periods, absence of any fibrous capsule coupled with the presence of mature adipocytes within the degrading gel. It is concluded that the chemical architecture of HYADD3 gel causes the de novo regeneration of soft tissue by recruitment of appropriate progenitor cells.
\end{abstract}

Keywords: chemical modification; adipose tissue; acellular; invivo; amidation; hyaluronan; inflammation; histology.

Reference to this paper should be made as follows: Rhodes, N.P., di Bartolo, C., Bellini, D. and Hunt, J.A. (XXXX) 'Induction of adipose tissue regeneration by chemically-modified hyaluronic acid', Int. J. Nano and Biomaterials, Vol. X, No. Y, pp.XXX-XXX.

Biographical notes: Please provide biographical notes for all authors to not more than 100 words. 


\section{Introduction}

There are a significant number of patients who suffer gross deficiency of soft tissue mass from trauma, hereditary disorders such as Poland syndrome (Fokin and Robicsek, 2002) and Romberg's disease (Finch and Dawe, 2003) and from treatment for tumours (e.g. mastectomy), accompanied by the potential for long term disfigurement. In addition to possible impaired mental health or low esteem, lack of subcutaneous adipose tissue generally leads to reduced limb movement. Patients who receive augmentation of their soft tissue for these conditions are commonly treated by transplantation of autologous mature adipose tissue. This strategy remains largely unchanged over many decades with often poor and unpredictable results (Billings and May, 1989). The reason for this relative lack of success is probably caused by the slow rate of revascularisation of the transplanted tissue (Nguyen et al., 1990) and the subsequent tissue ischaemia, leading to adipocyte resorption (Ersek, 1991), oil cysts and fibrous capsule formation. The most usual alternative therapies involve implantation of either natural or synthetic fillers, such as collagen whose main function is to replace lost volume. However, naturally derived fillers tend to shrink or are resorbed and synthetic alternatives can cause a chronic host response.

A surgical strategy based on tissue engineering principles whereby the injured site is directed to generate adipose soft tissue may provide a practical solution. In this scenario, it would be desirable to have an implantable biomaterial that would both, support the growth of adipocytes and actively induce neovascularisation of the graft. Hyualuronic acid occurs within the extracellular matrix compartment of most tissues and is well known for being actively angiogenic (Montesano et al., 1996). It is found within developing tissue and is implicated in tissue morphogenesis (Culty et al., 1992; Laurent and Fraser, 1992; Toole, 1981). A major stumbling block is that naturally occurring hyaluronan is a viscous fluid with limited retention and without significant mechanical properties. By chemical modification of the free carboxyl groups on the glucuronic acid component of the hyaluronan chain it has been clearly demonstrated that the solubility of the hyaluronan molecule is drastically reduced such that it is solid even after extended periods in aqueous environments (Iannace et al., 1992). Furthermore, by varying the degree of carboxyl modification, the degradation rate can be tailored to meet the requirements of the implantation site and application (Benedetti et al., 1993). It has been demonstrated in many studies that benzyl esterification of the carboxyl groups results in non-toxic degradation products (Cortivo et al., 1991; Hume et al., 1992), including free hyaluronan, which is highly angiogenic (Montesano et al., 1996). Hyaluronan-based materials that have been fashioned into non-woven fleeces, membranes and sponges have already been demonstrated as having efficacy when used as scaffolds in the reconstruction of skin (Zacchi et al., 1998), cartilage (Aigner et al., 1998) and bone (Gao et al., 2002; Solchaga et al., 2005). Moreover, it has been demonstrated that HYAFF-11-based sponges can support the differentiation of human adipocyte precursor cells in vitro (Halbleib et al., 2003). Strategies based on synthetic polymers, such as PLGA (Patrick et al., 1999) and PTFE (Kral and Crandall, 1999) have demonstrated severe limitations, including the mechanical properties of the scaffolds. Additionally, whilst the tissue remodelling effects of inflammation are usually regarded favourably in most post-surgical situations, the resultant phagocytic and trophic environment is unlikely to be ideal for a tissue engineering approach to soft tissue reconstruction. 
There are many clinical situations where an injectable scaffold would be more appropriate rather than an inflexible, solid scaffold, for example, non-uniform volumes or for filling small defects. A biomaterial has, therefore, been designed that is injectable but resistant to rapid degradation using amidation of the carboxyl groups rather than esterification. The hypothesis was that a gel scaffold that could support the growth of differentiating precursor cells in an angiogenic environment would be a good strategy for soft tissue reconstruction. The most desirable outcome would direct an inflammatory infiltration away from T-lymphocyte activation and towards cytokine secretion leading to preadipocyte infiltration, proliferation and differentiation. In this study, the ability of gels constructed from amidated hyaluronan, in unseeded form, to successfully form de novo soft tissue in vivo, without fibrotic capsule formation was investigated, and thereby their suitability as scaffolds for the tissue engineering of adipose tissue was evaluated.

\section{Materials and methods}

\subsection{Materials}

Gels were prepared by Fidia Advanced Biopolymers (Abano Terme, Italy) by partial chemical modification of hyaluronan by dodecyl-amidation of the carboxyl groups of the glucuronic acid components (HYADD3) (Figure 1). These were thermally sterilised prior to freeze drying. HYADD3 powder was dissolved in water at a concentration of $30 \mathrm{mg} / \mathrm{ml}$ prior to implantation (Figure 1(c)). Batch quality and safety were determined prior to implantation by performing standard endotoxin tests, and determining the concentration of residual solvents and the percentage of hyaluronan modification.

Figure 1 (a) Chemical structure of native hyaluronan showing carboxyl group that is amidated; (b) Dodecyl-amidated hyaluronan (HYADD3); (c) HYADD3 at working concentration

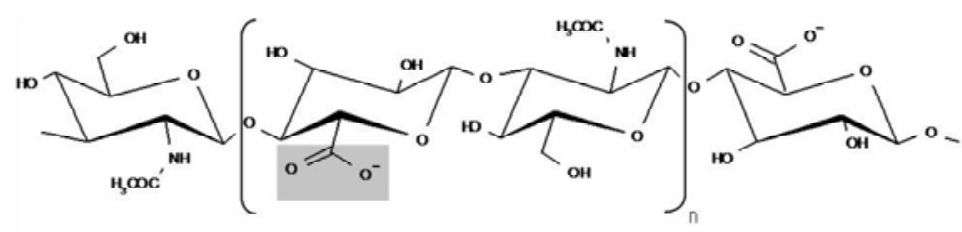

(a)

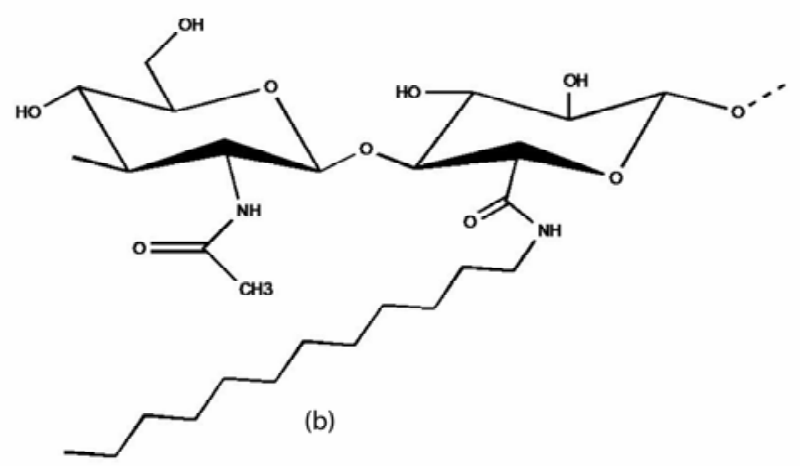


Figure 1 (a) Chemical structure of native hyaluronan showing carboxyl group that is amidated; (b) Dodecyl-amidated hyaluronan (HYADD3); (c) HYADD3 at working concentration (continued)

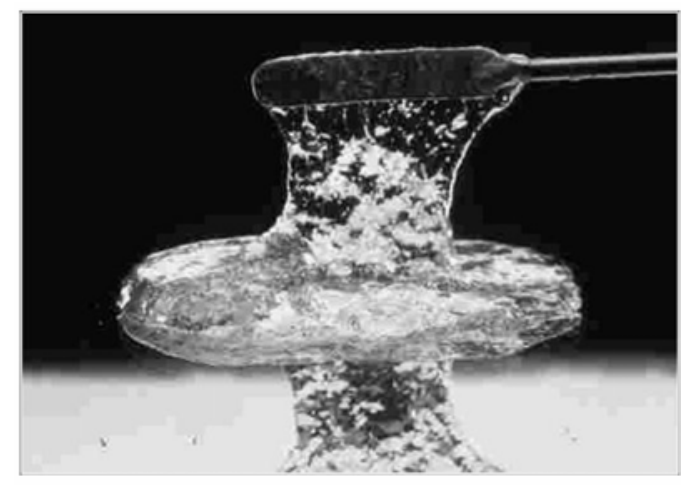

(c)

\subsection{Implantation}

HYADD3 gel was injected in $200 \mu \mathrm{l}$ aliquots through a $21 \mathrm{G}$ needle directly into the dorso-lumbar muscle of adult Wistar rats after creation of a small pocket using the injecting needle. Implantation time was 1, 2, 4, 8 and 12 weeks and was performed in both left and right sides of five rats for each time period. Each animal was sacrificed at the end of the implantation period. All surgery was performed in accordance with UK Home Office regulations.

\subsection{Characterisation of the host response}

Immediately after the sacrifice of experimental animals, gel implantation sites were explanted in situ with the surrounding tissue. The tissue was fixed in Paraformaldehyde Lysine Phosphate buffer (PLP fixative) and cold resin embedded in Glycol Methacrylate (GMA resin) preserving the antigenicity of the host tissue. Samples were sectioned to $4 \mu \mathrm{m}$ and analysed using the following tinctural histological stains: van Geison, von Kossa and haematoxylin \& eosin. Enzyme staining was performed for chloroacetate esterase staining. Immunohistochemistry was performed to specifically identify a number of cell types, subpopulations and activation states using the following mouse primary antirat monoclonal antibodies: ED1 (monocytes and immature macrophages), ED2 (CD163; differentiating macrophages), MHC-I, MHC-II (Major histocompatibility complexes), CD54 (ICAM-1), $\alpha-\beta$ receptor (T-cells), $\gamma-\delta$ receptor (T-cell subset), CD2 (mature T-cells), CD4 (helper/inducer T-cells), natural killer cells, B-cells, vimentin and TGF $\beta$, which were all obtained from Serotec (Oxford, UK). These were reacted with biotinylated rabbit antimouse immunoglobulin (DakoCytomation, Cambridge, UK) and colour developed red with alkaline phosphatase. PBS was used as the process negative control and the panel of antibodies was run together on two samples per full staining protocol to act as internal reference and isotype controls for the staining procedure. Positive staining was assessed by light microscopy; numbers of cells and their activation state were calculated using an Image Associates KS400 image analysis system 
(Cambridge, UK). Statistical significance was determined by ANOVA, utilising Tukey post hoc analysis with significance determined at the $95 \%$ confidence level using SPSS Version 10 (Chicago, IL, USA).

\section{Results}

There was little observed cell infiltration of the gels implanted intramuscularly after 1 week due to the high visco-elasticity of the gel, but this had changed and was much more apparent after 4 weeks (Figure 2(a) and (b)). Cells had infiltrated throughout the gel and into the centre of the gel mass, where the cells were actively producing extracellular matrix (Figure 2(c)). There was no appearance of gel degradation before 8 weeks and this was still only minimal after 12 weeks, HYADD3 demonstrated remarkable stability. The presence of neo-vessels was observed in all samples beyond the 2 week time period.

Figure 2 Gels implanted for 4 weeks in rats: (a) von kossa stained; (b) haematoxylin \& eosin stained, $\mathrm{V}$ denoting the presence of vessels; (c) van giesen stained (collagen positive)

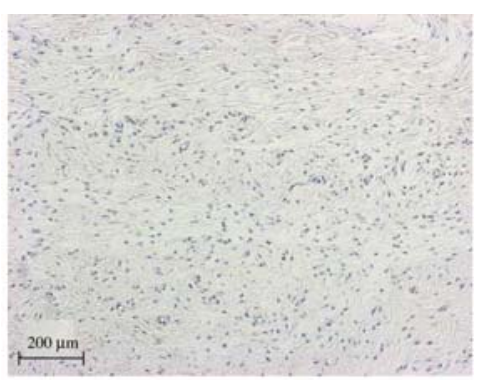

(a)

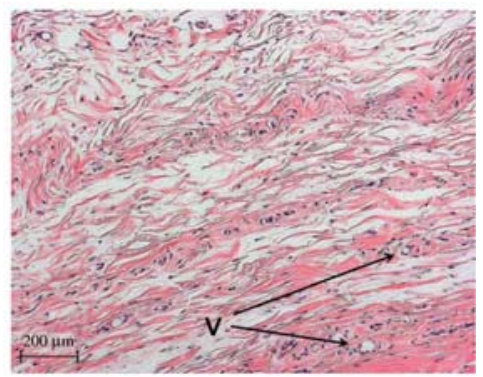

(b)

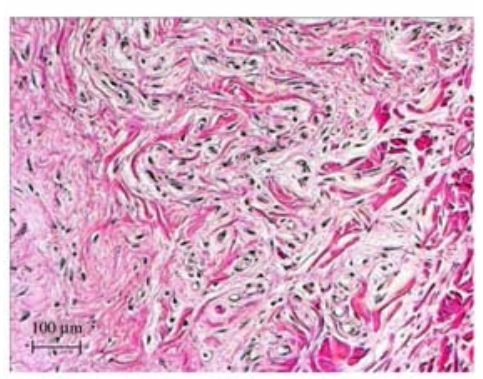

(c) 
Immunohistochemical analysis of the implants demonstrated very low overall levels of inflammation before 8 weeks (Figure 3), that reached a peak at 8 weeks and then declined thereafter. This was particularly so for the staining of the presence of immature and differentiating macrophages and the expression of MHC class I antigen. The positively stained cells were within both the gel and at the host-gel interface. After 12 weeks, the positive staining was confined to the interface only (Figure 4), with a reduction in the inflammatory response within the gel samples. Overall, MHCII expression was low for all implantation periods. There was discrete TGF $\beta$ expression, possibly associated with the vasculature but this was at a low level, indicative of an inflammatory response that was not progressing to a sustained chronic event, but rather, the macrophage infiltration was fairly benign and transient. Quantitative image analysis at 8 weeks demonstrated the dominance of macrophages in the inflammatory response profile (Figure 5) and verified the visual observations. The results clearly indicated that, of the cells within the gels, approximately $80-85 \%$ was macrophages and $10 \%$ were T-lymphocytes, with a similar percentage of B-lymphocytes. The T-cells that were present were generally $\mathrm{CD} 2$ positive (mature), with practically no lymphocyte infiltration until 8 weeks. There was significant vimentin expression within the gels. Quantitative analysis indicated that roughly half of the differentiated mature macrophages (ED2 positive) were vimentin positive, strongly suggesting the conversion of these macrophages to preadipocytes.

Figure 3 HYADD3 gel after 4 weeks intramuscular implantation in the rat, immunohistochemical staining for (a) differentiating macrophages; (b) T-lymphocytes; (c) MHC-I

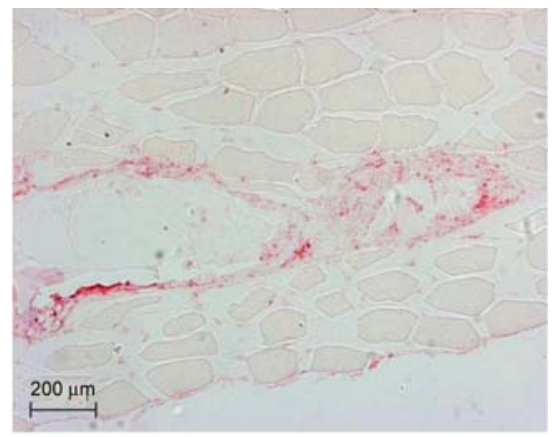

(a)

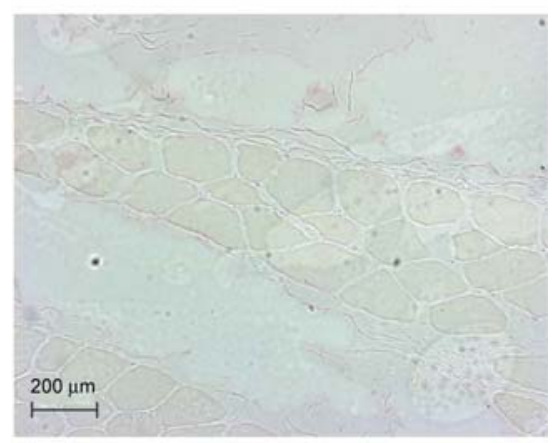

(b) 
Figure 3 HYADD3 gel after 4 weeks intramuscular implantation in the rat, immunohistochemical staining for (a) differentiating macrophages; (b) T-lymphocytes; (c) MHC-I (Continued)

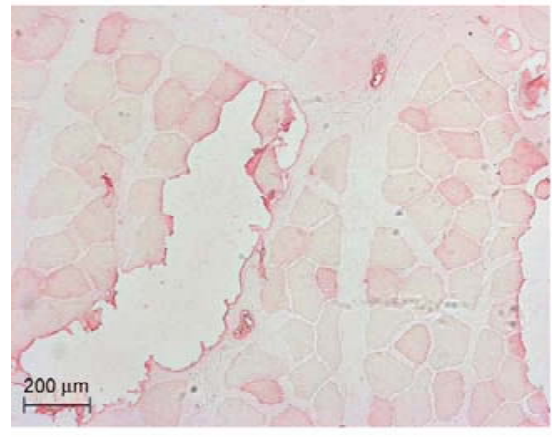

(c)

Figure 4 HYADD3 gel after 12 weeks intramuscular implantation in the rat, immunohistochemical staining for $(a, d)$ immature macrophages; (b, e) differentiating macrophages; (c, f) MHC-I. Lower row images: staining at the interface

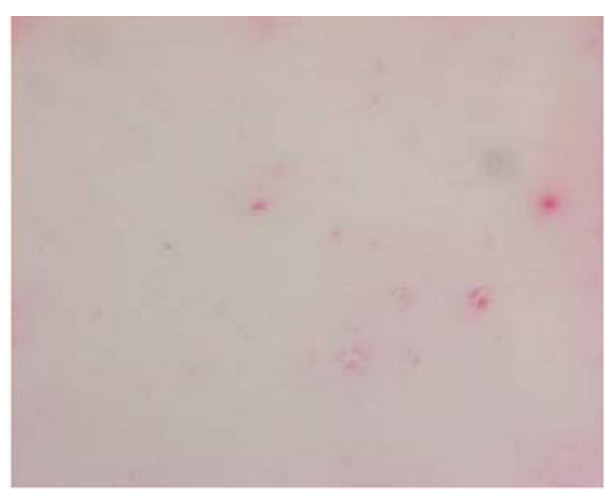

(a)

(b) 
Figure 4 HYADD3 gel after 12 weeks intramuscular implantation in the rat, immunohistochemical staining for (a,d) immature macrophages;

(b, e) differentiating macrophages; (c, f) MHC-I. Lower row images: staining at the interface (Continued)

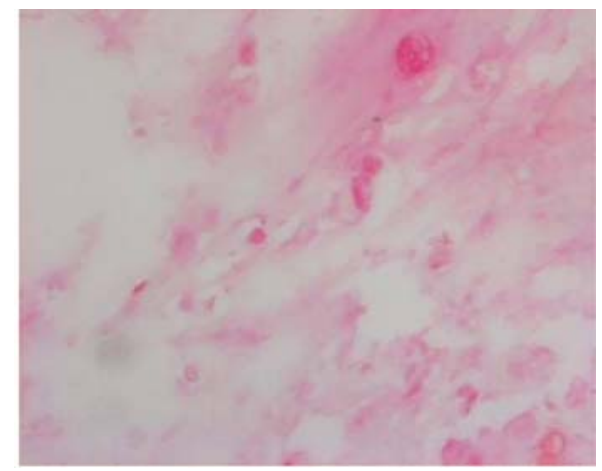

(c)

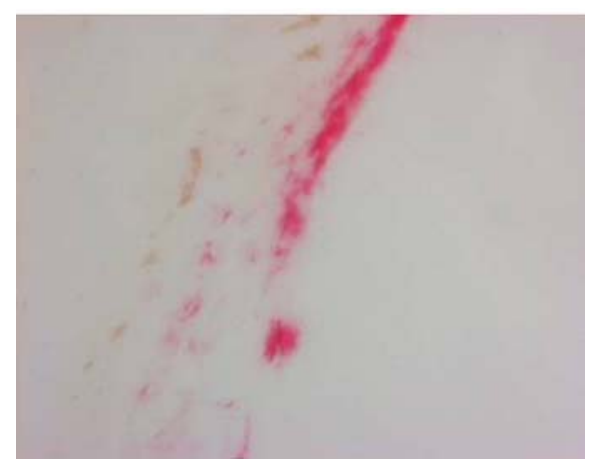

(d)

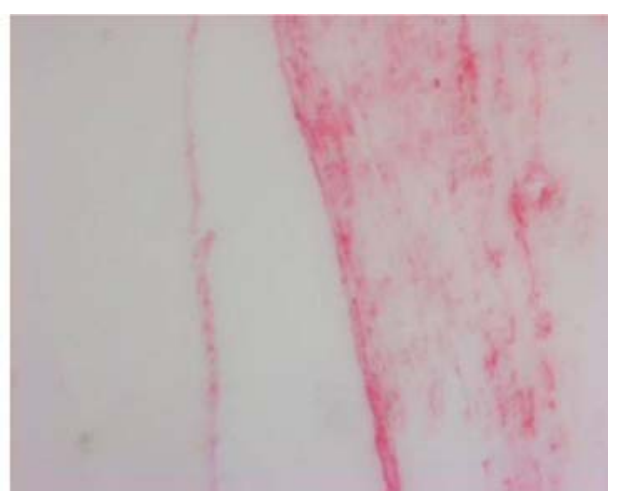

(e) 
Figure 4 HYADD3 gel after 12 weeks intramuscular implantation in the rat, immunohistochemical staining for (a, d) immature macrophages; (b, e) differentiating macrophages; (c, f) MHC-I. Lower row images: staining at the interface (Continued)

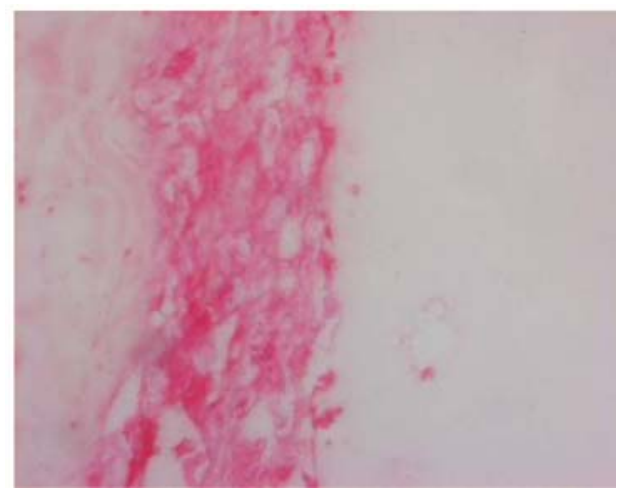

(f)

Figure 5 Numbers of cells positive for different markers, 8 weeks intramuscular implantation in rats, Mean \pm SEM, $n=10$

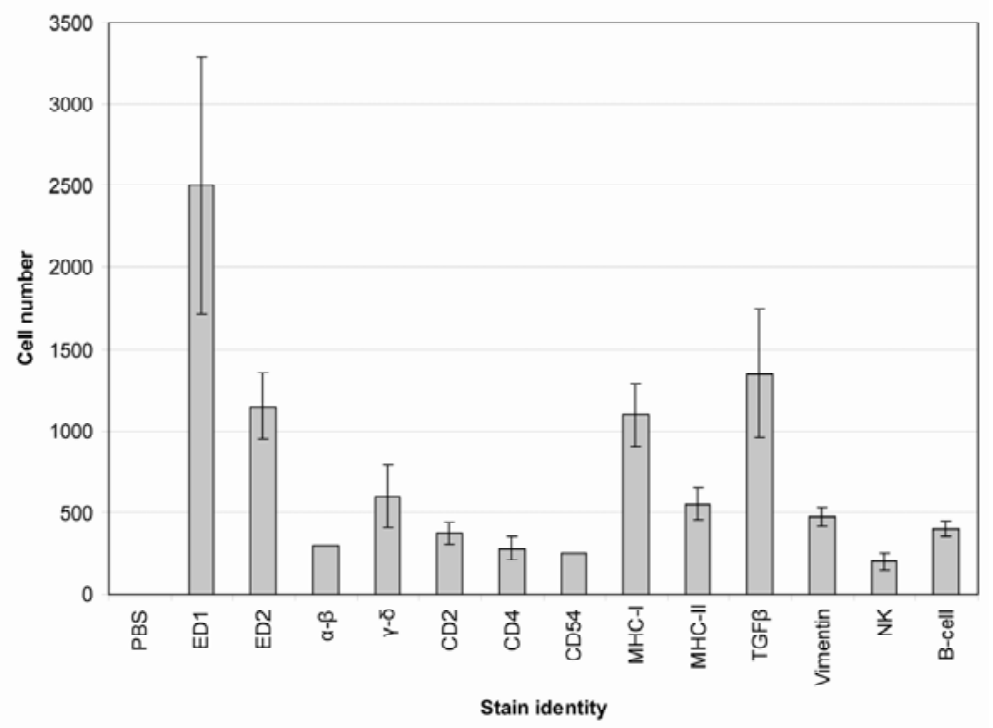

It was possible to observe the presence of mature adipocytes within all 12 week implants (Figure 6), the variable size indicating progression of fat droplet accumulation and so adipocyte development and maturation. Although the presence of a certain number of mature adipocytes can be put down to infiltration from the indigenous subdermal fat layer, many examples of gel without association with this layer containing adipocytes were observed, indicating de novo adipogenesis. Tissue integration with the host was excellent, with no fibrous layer evident at the interface between the gel and the indigenous tissue (Figure 6). 
Figure 6 HYADD3 gel after 12 weeks implantation, haematoxylin \& eosin stained, A denoting the presence of mature adipocytes

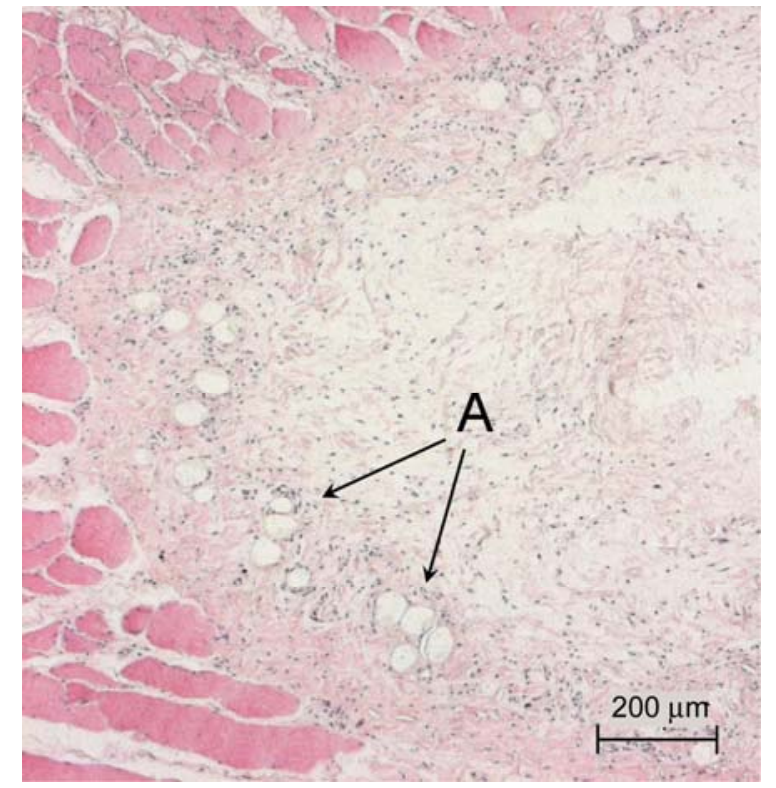

\section{Discussion}

Tissue engineering strategies have been developed for many tissues and these are beginning to reach clinic, most notably in applications related to the regeneration of connective tissues. The difficulties in developing successful tissue engineering strategies often relate to the conversion of in vitro observations of cell proliferation and differentiation of precursor-laden scaffolds into similar tissue when placed into an implant site. Many histological studies of implanted biomaterials, medical devices and tissue engineering constructs fail to take into account the nature of the observed inflammatory response, and report merely its location and magnitude. The secretion of various cytokines by macrophages and other infiltrating cells is intrinsic to the development of a microenvironment which will control the successful maturation of either implanted or migrating cells into the desired tissue phenotype. In this way, the scaffold can be seen to be central to the development of this microenvironment by virtue of its surface chemistry and nanotopography, rather than being simply a passive structure.

Many researchers have studied the commercial aspects of tissue engineering and regenerative medicine, and concluded that the market penetration of such products (TEPs) is a tiny fraction of the potential market (Landesbank Baden-Württemberg Equity Research, 2001; Russell and Cross, 2001). Whilst much work is required to demonstrate the increased efficacy of TEPs combined with satisfactory safety in comparison to conventional medical devices, it is still likely that companies are nervous about the inherent variability of the cost basis of cellular TEPs, which has led to the lack of investment in the technology by large pharmaceutical companies. It is much more likely that commercial availability of regenerative medicine therapies would be achieved if 
acellular strategies were available, achieving a predictable cost base. It is important, therefore, that biomaterials possessing the ability to induce the regeneration of different tissues by virtue of their chemical and topographical architecture are developed.

The results of this study strongly suggest that HYADD3 gel has the ability to induce the regeneration of adipose tissue without the implantation of cells. Not only are mature and maturing adipocytes observed within gels implanted into rats, but macrophages expressing vimentin are visible after 4 weeks. Although macrophages are generally associated with phagocytosis and inflammation, it is now recognised that they transdifferentiate into adipocytes (Charrière et al., 2003; Saillan-Barreau et al., 2003). Furthermore, vimentin expression has long been associated with differentiating human and rat preadipocytes (Teichert-Kuliszewska et al., 1996). It was clear from the results that the inflammatory response due to the gel implantation was not intense, but was nevertheless present. It is often noted that adipocyte differentiation is reduced in inflammatory milieu whereas it is well recognised that there is a close association between angiogenesis and adipogenesis (Hausman and Richardson, 2004), angiogenesis being profoundly augmented by inflammatory conditions. Clearly, the precise nature of the inflammatory response and its intensity is of key importance in this regard.

Neo-vessels were observed from early time periods, demonstrating an angiogenic response due to the presence of the gel. We propose that inflammatory-cell infiltration without high levels of activation-related expression (e.g. MHCII) linked with increased angiogenesis were responsible for in vivo adipogenesis. It is this combination of factors that was observed within the implanted gels (HYADD3), with low levels of lymphocyte infiltration and activation, coupled with differentiating macrophages.

The precise nature of the biochemical signalling in response to the implantation of HYADD3 will be studied in future experiments, including the sensitivity of that response to the amide chain length, which has an impact on gel degradation and therefore release of angiogenic hyaluronic monomers.

\section{Conclusion}

This study demonstrated that the implantation of HYADD3 gel in rats induces low overall levels of inflammation. Infiltration was predominantly due to macrophages, half of the mature population of which were expressing vimentin indicating development into preadipocytes. De novo adipogenesis was indicated at 12 weeks, suggesting that HYADD3 could be implanted without cells to induce the regeneration of soft tissue.

\section{Acknowledgement}

Funding for research in this study was provided by the European Commission under the Competitive and Sustainable Growth Programme of the 5th Framework, contract number G5RD-CT-1999-00111. Parts of the research were performed as part of the UK Interdisciplinary Research Collaboration in Tissue Engineering for which funds were provided by the BBSRC, MRC and EPSRC. Provision of an Advanced Research Fellowship for Nicholas Rhodes by EPSRC is gratefully acknowledged. 


\section{References}

Aigner, J., Tegeler, J., Hutzler, P., Campoccia, D., Pavesio, A., Hammer, C., Kastenbauer, E. and Naumann, A. (1998) 'Cartilage tissue engineering with novel nonwoven structured biomaterial based on hyaluronic acid benzyl ester', Journal of Biomedical Materials Research, Vol. 42, pp.172-181.

Benedetti, L., Cortivo, R., Berti, T., Berti, A., Pea, F., Mazzo, M., Moras, M. and Abatangelo, G. (1993) 'Biocompatibility and biodegradation of different hyaluronan derivatives (HYAFF) implanted in rats', Biomaterials, Vol. 14, pp.1154-1160.

Billings, E. and May, J.W. (1989) 'Historical review and present status of free fat graft autotransplantation in plastic and reconstructive surgery', Plastic and Reconstructive Surgery, Vol. 83, pp.368-381.

Charrière, G., Cousin, B., Arnaud, E., André, M., Bacou, F., Pénicaud, L. and Casteilla, L. (2003) 'Preadipocyte conversion to macrophage - evidence of plasticity', Journal of Biological Chemistry, Vol. 278, pp.9850-9855.

Cortivo, R., Brun, P., Rastrelli, A. and Abatangelo, G. (1991) 'In vitro studies on biocompatibility of hyaluronic-acid esters', Biomaterials, Vol. 12, pp.727-730.

Culty, M., Nguyen, H.A. and Underhill, C.B. (1992) 'The hyaluronan receptor (CD44) participates in the uptake and degradation of hyaluronan', Journal of Cell Biology, Vol. 116, pp.1055-1062.

Ersek, R.A. (1991) 'Transplantation of purified autologous fat - a 3-year follow-up is disappointing', Plastic and Reconstructive Surgery, Vol. 87, pp.219-227.

Finch, G.D. and Dawe, C.J. (2003) 'Hemiatrophy', Journal of Pediatric Orthopaedics, Vol. 23, pp.99-101.

Fokin, A.A. and Robicsek, F. (2002) 'Poland's syndrome revisited', Annals of Thoracic Surgery, Vol. 74, pp.2218-2225.

Gao, R.Z., Dennis, J.E., Solchaga, L.A., Goldberg, V.M. and Caplan, A.I. (2002) 'Repair of osteochondral defect with tissue-engineered two-phase composite material of injectable calcium phosphate and hyaluronan sponge', Tissue Engineering, Vol. 8, pp.827-837.

Halbleib, M., Skurk, T., de Luca, C., von Heimburg, D. and Hauner, H. (2003) 'Tissue engineering of white adipose tissue using hyaluronic acid-based scaffolds. I: in vitro differentiation of human adipocyte precursor cells on scaffolds', Biomaterials, Vol. 24, pp.3125-3132.

Hausman, G.J. and Richardson, R.L. (2004) 'Adipose tissue angiogenesis', Journal of Animal Science, Vol. 82, pp.925-934.

Hume, L., Kyyronen, K., Benedetti, L., Stella, V. and Topp, E. (1992) 'Drug delivery devices from hyaluronic-acid esters - in vivo applications', Abstracts and Papers of the American Chemical Society, Vol. 203, p.67.

Iannace, S., Ambrosio, L., Nicolais, L., Rastrelli, A. and Pastorello, A. (1992) 'Thermomechanical properties of hyaluronic acid-derived products', Journal of Materials Science - Materials in Medicine, Vol. 3, pp.59-64.

Kral, J.G. and Crandall, D.L. (1999) 'Development of a human adipocyte synthetic polymer scaffold', Plastic and Reconstructive Surgery, Vol. 104, pp.1732-1738.

Landesbank Baden-Württemberg Equity Research (2001) Tissue Engineering, Landesbank Baden-Württemberg Equity Research, p.62.

Laurent, T.C. and Fraser, J.R.E. (1992) 'Hyaluronan', Federation of American Societies for Experimental Biology Journal, Vol. 6, pp.2397-2404.

Montesano, R., Kumar, S., Orci, L. and Pepper, M.S. (1996) 'Synergistic effect of hyaluronan oligosaccharides and vascular endothelial growth factor on angiogenesis in vitro', Laboratory Investigation, Vol. 75, pp.249-262.

Nguyen, A., Pasyk, K.A., Bouvier, T.N., Hassett, C.A. and Argenta, L.C. (1990) 'Comparative study of survival of autologous adipose tissue taken and transplanted by different techniques', Plastic and Reconstructive Surgery, Vol. 85, pp.378-386. 
Patrick, C.W., Chauvin, P.B., Hobley, J. and Reece, G.P. (1999) 'Preadipocyte seeded PLGA scaffolds for adipose tissue engineering', Tissue Engineering, Vol. 2, pp.139-151.

Russell, J. and Cross, S. (2001) 'Commercial prospects for tissue engineering', Business Intelligence Program, SRI Consulting, p.15.

Saillan-Barreau, C., Cousin, B., André, M., Villena, P., Casteilla, L. and Pénicaud, L. (2003) 'Human adipose cells as candidates in defense and tissue remodeling phenomena', Biochemical and Biophysical Research Communications, Vol. 309, pp.502-505.

Solchaga, L.A., Temenoff, J.S., Gao, J.Z., Mikos, A.G., Caplan, A.I. and Goldberg, V.M. (2005) 'Repair of osteochondral defects with hyaluronan- and polyester-based scaffolds', Osteoarthritis Cartilage, Vol. 13, pp.297-309.

Teichert-Kuliszewska, K., Hamilton, B.S., Roncari, D.A., Kirkland, J.L., Gillon, W.S., Deitel, M. and Hollenberg, C.H. (1996) 'Increasing vimentin expression associated with differentiation of human and rat preadipocytes', International Journal of Obesity Related Metabolic Disorders, Vol. 20, Suppl. 3, pp.S108-113.

Toole, B.P. (1981) 'GAGs in morphogenesis', in E.D. Hay (Ed). Cell Biology of the Extracellular Matrix, New York, USA: Plenum, pp.259-294.

Zacchi, V., Soranzo, C., Cortivo, R., Radice, M., Brun, P. and Abatangelo, G. (1998) 'In vitro engineering of human skin-like tissue', Journal of Biomedical Materials Research, Vol. 40, pp.187-194. 